University of Wollongong

Research Online

Faculty of Engineering - Papers (Archive)

Faculty of Engineering and Information

Sciences

2003

\title{
Effect of carbon nanotube doping on critical current density of MgB2 superconductor
}

SX. Dou

University of Wollongong, shi@uow.edu.au

W. K. Yeoh

University of Wollongong

J. Horvat

University of Wollongong, jhorvat@uow.edu.au

M. Ionescu

University of Wollongong, mionescu@uow.edu.au

Follow this and additional works at: https://ro.uow.edu.au/engpapers

Part of the Engineering Commons

https://ro.uow.edu.au/engpapers/98

\section{Recommended Citation}

Dou, S X.; Yeoh, W. K.; Horvat, J.; and lonescu, M.: Effect of carbon nanotube doping on critical current density of MgB2 superconductor 2003.

https://ro.uow.edu.au/engpapers/98

Research Online is the open access institutional repository for the University of Wollongong. For further information contact the UOW Library: research-pubs@uow.edu.au 


\title{
Effect of carbon nanotube doping on critical current density of $\mathrm{MgB}_{2}$ superconductor
}

\author{
S. X. Dou, W. K. Yeoh, J. Horvat, ${ }^{\text {a) }}$ and M. Ionescu \\ Institute for Superconducting and Electronic Materials, University of Wollongong, Northfields Avenue, \\ Wollongong, New South Wales 2522, Australia
}

(Received 8 August 2003; accepted 23 October 2003)

\begin{abstract}
The effect of doping $\mathrm{MgB}_{2}$ with carbon nanotubes on transition temperature, lattice parameters, critical current density and flux pinning was studied for $\mathrm{MgB}_{2-x} \mathrm{C}_{x}$ with $x=0,0.05,0.1,0.2$, and 0.3. The carbon substitution for B was found to enhance $J_{c}$ in magnetic fields but depress $T_{c}$. The depression of $T_{c}$, which is caused by the carbon substitution for B, increases with an increasing doping level, sintering temperature, and duration. By controlling the extent of the substitution and addition of carbon nanotubes we can achieve the optimal improvement on critical current density and flux pinning in magnetic fields while maintaining the minimum reduction in $T_{c}$. Under these conditions, $J_{c}$ was enhanced by two orders of magnitude at $8 \mathrm{~T}$ and $5 \mathrm{~K}$ and $7 \mathrm{~T}$ and $10 \mathrm{~K}$. $J_{c}$ was more than $10000 \mathrm{~A} / \mathrm{cm}^{2}$ at $20 \mathrm{~K}$ and $4 \mathrm{~T}$ and $5 \mathrm{~K}$ and $8.5 \mathrm{~T}$, respectively. (c) 2003 American Institute of Physics. [DOI: 10.1063/1.1634378]
\end{abstract}

The effect of C-doping on superconductivity in a $\mathrm{MgB}_{2}$ compound has been studied by several groups. ${ }^{1-8}$ The results on C solubility and the effect of C-doping on $T_{c}$ reported so far vary significantly due to the precursor materials, fabrication techniques, and processing conditions used. It appears that lower sintering temperatures and short sintering times result in an incomplete reaction and hence lower $\mathrm{C}$ solubility in $\mathrm{MgB}_{2}$. Ribeiro et al. used $\mathrm{Mg}$ and $\mathrm{B}_{4} \mathrm{C}$ as precursors to synthesize C-doped $\mathrm{MgB}_{2}$ by sintering at $1200{ }^{\circ} \mathrm{C}$ for $24 \mathrm{~h} .{ }^{9}$ A neutron diffraction study confirmed that the most likely solubility of $\mathrm{C}$ in $\mathrm{MgB}_{2}$ is up to around $10 \%$ of $\mathrm{C}$ in the boron sites, resulting in a large drop of both $T_{c}$ and the $a$-axis lattice parameter. ${ }^{10}$ Recently, Lee et al. synthesised C-doped single crystalline $\mathrm{MgB}_{2}$ at high pressure (5 GPa) and high temperature $\left(1600{ }^{\circ} \mathrm{C}\right)$, obtaining the $\mathrm{C}$ solubility of $15 \%$ at the boron sites and $T_{c}$ depression to below $3 \mathrm{~K} .{ }^{11}$ All these studies on $\mathrm{C}$ doping into $\mathrm{MgB}_{2}$ have only focused on the effect on superconductivity. From the applications point of view, the effect of $\mathrm{C}$ doping on the flux pinning properties is crucially important. The author's group has reported a significant improvement in $J_{c}(H)$ and $H_{\text {irr }}$ in $\mathrm{MgB}_{2}$ through nano-SiC doping. ${ }^{12}$ Recently, the effects of $\mathrm{C}$ doping on the flux pinning and critical current density in $\mathrm{MgB}_{2}$ has been studied using amorphous carbon ${ }^{13}$ and diamond, ${ }^{14}$ both showing improvement of $J_{c}$ at elevated magnetic fields. Wei et al. have studied the superconductivity of $\mathrm{MgB}_{2}$-carbon nanotube composites. ${ }^{15}$ However, the effect of carbon nanotube doping on critical current density and flux pinning has not been reported. Among various carbon precursors, carbon nanotubes are particularly interesting as their special geometry (high aspect ratio and nanometer diameter) may induce more effective pinning centers compared to other carboncontaining precursors. In this letter we report the results on control of the extent of carbon nanotube substitution and addition to achieve an enhancement of critical current density and flux pinning by two orders of magnitude in magnetic fields.

${ }^{a)}$ Electronic mail: jhorvat@uow.edu.au
Polycrystalline samples of $\mathrm{MgB}_{2-x} \mathrm{C}_{x}$ were prepared through a reaction in-situ process. ${ }^{16,17}$ High purity powders of magnesium (99\%), amorphous boron (99\%) and multiwalled carbon nanotubes of 20-30 nm diameter were weighed out according to the nominal atomic ratio of $\mathrm{MgB}_{2-x} \mathrm{C}_{x}$ with $x=0,0.05,0.1,0.2,0.3$, and well-mixed through grinding. We stress that the values for $x$ are the nominal values throughout the paper, and the actual substitution of C for B will be shown to be less than these values. The powders were pressed into pellets of $10 \mathrm{~mm}$ in diameter and $3 \mathrm{~mm}$ in thickness using a hydraulic press. The pellets were sealed in $\mathrm{Fe}$ tubes, then heat treated at 700 to $1000{ }^{\circ} \mathrm{C}$ for 10-120 min in flowing high purity Ar. This was followed by a furnace cooling to room temperature. An undoped sample was also made under the same conditions for use as a reference sample. The phase and crystal structure of all the samples was obtained from x-ray diffraction (XRD) patterns using a MAC Science MX03 diffractometer with $\mathrm{Cu} K \alpha$ radiation. Si powder was used as an internal standard to calculate the lattice parameters. The grain morphology and microstructure were also examined by a scanning electron microscope (SEM) and a transmission electron microscope (TEM).

The magnetization was measured over a temperature range of 5-30 K using a Physical Property Measurement System (PPMS, Quantum Design) in a time-varying magnetic field with sweep rate $50 \mathrm{Oe} / \mathrm{s}$ and amplitude $8.5 \mathrm{~T}$. Bar-shaped samples with a size of $4 \times 3 \times 0.5 \mathrm{~mm}^{3}$ were cut from each pellet for magnetic measurements. The magnetic measurements were performed by applying the magnetic field parallel to the longest sample axis. The magnetic $J_{c}$ was calculated from the height of the magnetization loop $\Delta M$ using the Bean model: $J_{c}=20 \Delta M /[a(1-a / 3 b)]$, with $a$ and $b$ as the dimensions of the sample perpendicular to the direction of applied magnetic field and $a<b . J_{c}$ versus the magnetic field has been measured up to $8.5 \mathrm{~T}$. The low field $J_{c}$ below $10 \mathrm{~K}$ could not be measured due to flux jumping. The $T_{c}$ was determined by measuring the real part of the ac susceptibility at a frequency of $117 \mathrm{~Hz}$ and an external mag- 


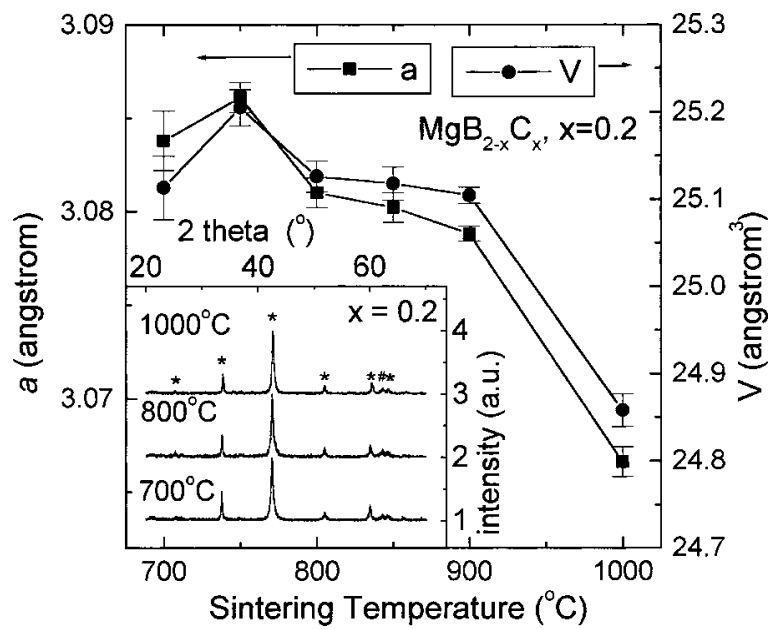

FIG. 1. The variation of lattice parameter $a$ and the unit cell volume of $\mathrm{MgB}_{2-x} \mathrm{C}_{x}$, with nominal $x=0.2$, with sintering temperature. The carbon was in the form of multiwalled carbon nanotubes. Inset: an XRD pattern for carbon nanotube doped $\mathrm{MgB}_{2}$, sintered at temperatures as indicated in the figure. Symbols* and \# indicate the XRD peaks for $\mathrm{MgB}_{2}$ and $\mathrm{MgO}$, respectively.

netic field of 0.1 Oe. $T_{c}$ was defined as the onset of the diamagnetism.

Figure 1 shows lattice parameter $a$, and unit cell volume versus sintering temperature for the sample doped at $x$ $=0.2$. The $a$ axis decreases monotonically with increasing sintering temperature. The $c$ axis varies very little with the sintering temperature and consequently the volume changes in a similar manner as $a$ (Fig. 1). The decrease of the $a$-axis is an indication of the boron substitution for carbon. The decrease of the $a$ axis is more pronounced at temperatures above $900{ }^{\circ} \mathrm{C}$, because of the enhanced carbon substitution at these temperatures, which is consistent with several recent papers. ${ }^{10,11}$ However, the substitution reaction in the present work is far from completion, even at sintering temperature of $1000^{\circ} \mathrm{C}$, in comparison with those treated at a higher temperature $\left(1600^{\circ} \mathrm{C}\right)$ and high pressure. ${ }^{11}$ Thus, we achieved a condition of partial substitution of $\mathrm{C}$ for $\mathrm{B}$ and the addition of a majority of $\mathrm{C}$, which may react with $\mathrm{B}$ to form $\mathrm{BC}$, as detected using EELS, ${ }^{18}$ or stay as carbon nanotubes. The inset to Fig. 1 shows the XRD data for carbon nanotube doped samples, sintered at three different temperatures. The sample was a well-developed $\mathrm{MgB}_{2}$ phase, with only a small amount of $\mathrm{MgO}$ present, similar to other high-quality $\mathrm{MgB}_{2}$ samples. ${ }^{12,13,17}$

Figure 2 shows the transition temperature $\left(T_{c}\right)$ for the doped and undoped samples determined by ac susceptibility measurements. The $T_{c}$ onset for the undoped sample is $\sim 38$ $\mathrm{K}$. For the sample doped at $x=0.2(10 \%$ of B) and sintered for a fixed period of $30 \mathrm{~min}$, the $T_{c}$ decreases with increasing sintering temperature. $T_{c}$ reaches $31 \mathrm{~K}$ for the sintering temperature of $1000^{\circ} \mathrm{C}$. This indicates that the extent of the $\mathrm{C}$ substitution reaction increases with increasing sintering temperature, resulting in $T_{c}$ depression, which is consistent with the recent reports. ${ }^{10,11}$ The effect of doping level on $T_{c}$ was also studied at a fixed sintering temperature of $800^{\circ} \mathrm{C}$ for 30 $\min$. For this sintering temperature, $T_{c}$ only drops slightly, $2.0 \mathrm{~K}$ at a $\mathrm{C}$ doping level of $x=0.3$ ( $15 \% \mathrm{C}$ doping). These results suggest that only a small amount of $\mathrm{C}$ nanotube powder was substituted in the B position in the samples sintered

Downloaded 19 Jun 2006 to 130.130.37.6. Redistribution subject

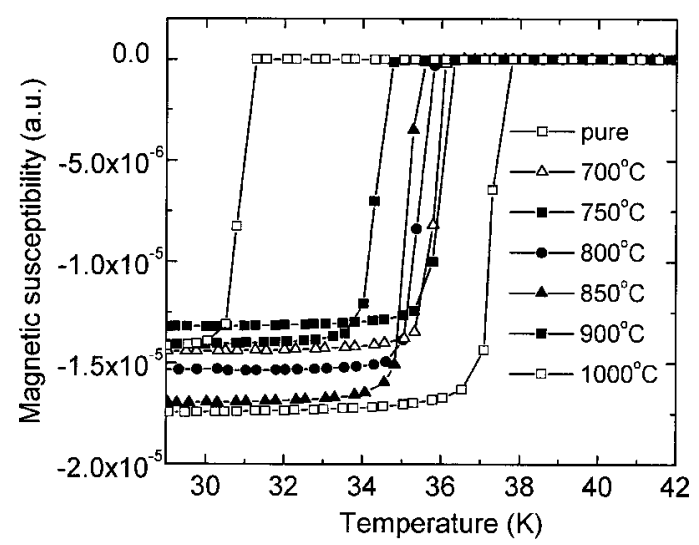

FIG. 2. Magnetic ac susceptibility as a function of temperature for $\mathrm{MgB}_{2-x} \mathrm{C}_{x}$ sintered at different temperatures for $30 \mathrm{~min}$. Carbon added was in the form of multiwalled carbon nanotubes.

at low temperature and a short period, consistent with small crystal lattice contraction. In order to improve the $J_{c}$ at higher temperature, such as $20 \mathrm{~K}$, it is essential to maintain high $T_{c}$. The above results indicate that by manipulating the processing parameters we could control the $T_{c}$ while achieving a high level of $\mathrm{C}$ inclusion into $\mathrm{MgB}_{2}$ sample, up to $10 \%$ of B. Because such $\mathrm{C}$ inclusion has little effect on $T_{c}$, the partial substitution of boron for carbon and the partial addition of nanocarbon particles into a $\mathrm{MgB}_{2}$ matrix may enhance flux pinning within a wide range of temperatures.

Figure 3 shows the $J_{c}(\mathrm{H})$ curves at 5 and $20 \mathrm{~K}$ for the samples of $\mathrm{MgB}_{2-x} \mathrm{C}_{x}$, where $x=0,0.05,0.1,0.2$, and 0.3 are the nominal values for $\mathrm{C}$ content, with all the samples sintered at $800^{\circ} \mathrm{C}$ for $30 \mathrm{~min}$. All the $J_{c}(\mathrm{H})$ curves for doped samples have a higher $J_{c}$ than the undoped sample at high fields. The sample doped with $10 \%$ of carbon nanotubes $(x$ $=0.2$ ) gives the best $J_{c}$ at high fields: $J_{c}$ increases by a factor of 45 at $5 \mathrm{~K}$ for the field of $8 \mathrm{~T}$, and at $20 \mathrm{~K}$ for the field of $5 \mathrm{~T}$, as compared to the undoped sample. At a higher doping level $(x=0.3)$, although the $J_{c}$ in low-field regime was depressed, the rate of $J_{c}$ drop is much slower than for all other samples, clearly indicating strong flux pinning induced by the $\mathrm{C}$ nanotube doping.

Figure 4 shows the $J_{c}(\mathrm{H})$ curves at 5 and $20 \mathrm{~K}$ for the $\mathrm{MgB}_{1.8} \mathrm{C}_{0.2}$ sample sintered at temperatures from $700{ }^{\circ} \mathrm{C}-$

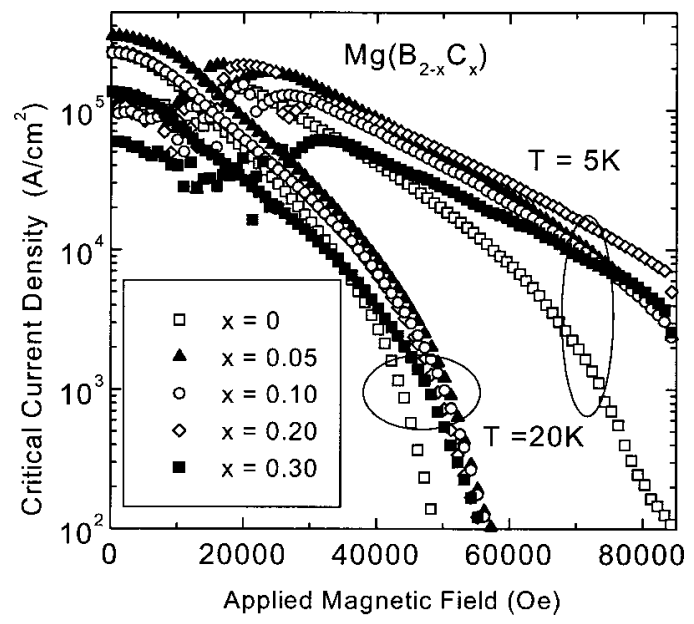

FIG. 3. Critical current density as a function of the magnetic field at 5 and $20 \mathrm{~K}$ for a different doping level of multi-walled carbon nanotubes.

AIP license or copyright, see http://apl.aip.org/apl/copyright.jsp 


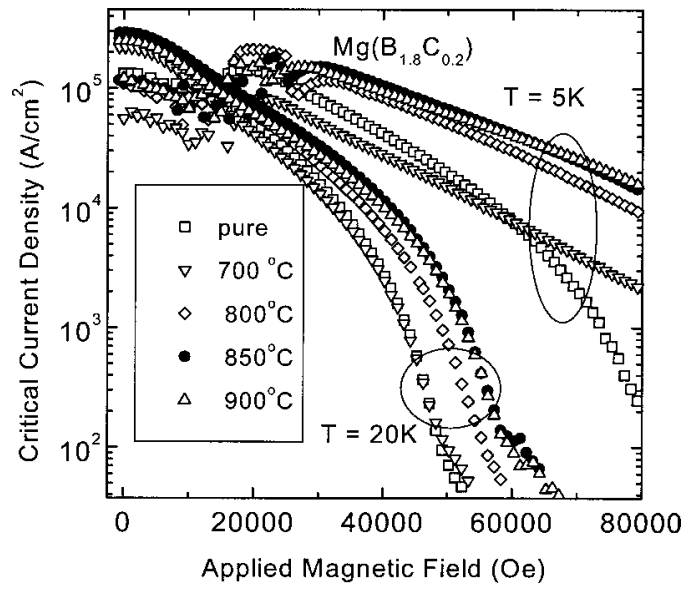

FIG. 4. The critical current density as a function of magnetic field at 5 and $20 \mathrm{~K}$ for $\mathrm{MgB}_{1.8} \mathrm{C}_{0.2}$, sintered at different temperatures for $30 \mathrm{~min}$. The carbon was in the form of multiwalled carbon nanotubes.

$1000^{\circ} \mathrm{C}$ for $30 \mathrm{~min}$. For comparison, the $J_{c}(\mathrm{H})$ curve for the undoped sample, $\mathrm{MgB}_{2}$ sintered at $800^{\circ} \mathrm{C}$ is included. It is noted that the sintering temperature has a significant effect on the $J_{c}$ performance in the field. A general trend is such that the $J_{c}(\mathrm{H})$ characteristic is improved with increasing sintering temperature. Although the sample sintered at $1000^{\circ} \mathrm{C}$ has lower $J_{c}$ values in the low-field regime, its $J_{c}(\mathrm{H})$ curve crosses over the $J_{c}(\mathrm{H})$ for the other samples in higher fields. As a higher sintering temperature promotes the $\mathrm{C}$ substitution reaction for $\mathrm{B}$, the improved field dependence of $J_{c}$ measured at lower temperatures is clearly attributable to the $\mathrm{C}$ substitution. However, because $\mathrm{C}$ substitution depresses $T_{c}$, the $J_{c}(\mathrm{H})$ behavior for samples processed at high temperatures deteriorates above $20 \mathrm{~K}$. Thus, it is important to control the extent of $\mathrm{C}$ nanotube substitution and addition to achieve the best combination of the substitution-induced flux pinning and $\mathrm{C}$ nanotube additive pinning.

Figure 5 compares the $J_{c}(\mathrm{H})$ for carbon nanotubes and nano-C particle ${ }^{13}$ doped $\mathrm{MgB}_{2}$ at 5 and $20 \mathrm{~K}$. It is noted that

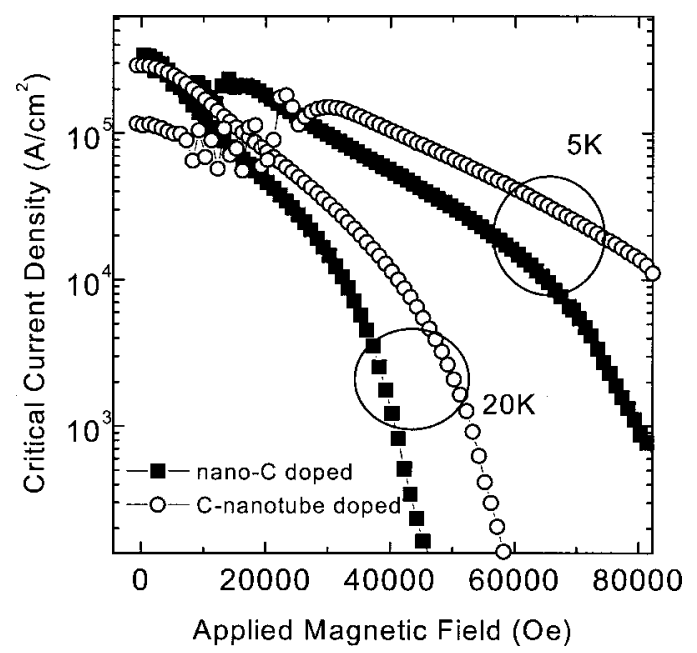

FIG. 5. The field dependence of $J_{c}$ for $10 \%$ nano-C doped (solid symbols) and $10 \%$ carbon nanotube doped (open symbols) $\mathrm{MgB}_{2}$ at 5 and $20 \mathrm{~K}$. This doping level corresponds to the nominal $x=0.2$. The sweep rate of the field was $50 \mathrm{Oe} / \mathrm{s}$. the carbon nanotubes produced a stronger enhancement of $J_{c}$ than the nano-C particles. The reasons for $\mathrm{C}$ nanotube doping being far better than $\mathrm{C}$ nanoparticle doping can be explained as follows. The optimum doping level for nano-C particles is $x=0.05(2.5 \%$ of $\mathrm{B})$ while this level increases to $x=0.2$ (10\% of B) for $\mathrm{C}$ nanotube doping. This indicates that the superconductivity of $\mathrm{MgB}_{2}$ shows a higher tolerance to $\mathrm{C}$ nanotubes than $\mathrm{C}$ nanoparticles. Consequently, there can be a higher concentration of nanoinclusions in the $\mathrm{C}$ nanotube doped sample than in the $\mathrm{C}$ nanoparticle doped ones, for the same value of $T_{c}$. Furthermore, the special geometry of $\mathrm{C}$ nanotubes is desirable for effective pinning.

In summary, the effect of $\mathrm{C}$ nanotube doping on lattice parameters, $T_{c}, J_{c}$ and flux pinning in $\mathrm{MgB}_{2}$ was investigated under a wide range of processing conditions. It was found that the substitution of the $\mathrm{C}$ nanotube for $\mathrm{B}$ enhances the flux pinning but depresses $T_{c}$. By controlling the processing parameters an optimized $J_{c}(\mathrm{H})$ performance is achieved under a partial $\mathrm{C}$ substitution and $\mathrm{C}$ nanoaddition. Under these conditions, $J_{c}$ was enhanced by two orders of magnitude at $8 \mathrm{~T}$ and $5 \mathrm{~K}$, and at $7 \mathrm{~T}$ and $10 \mathrm{~K}$. The $J_{c}$ was more than $10000 \mathrm{~A} / \mathrm{cm}^{2}$ at $20 \mathrm{~K}$ in the field of $4 \mathrm{~T}$ and at 5 $\mathrm{K}$ in the field of $8.5 \mathrm{~T}$, respectively. Carbon nanotube inclusions and $\mathrm{C}$ substitution for $\mathrm{B}$ are proposed to be responsible for the enhancement of flux pinning in high fields.

The authors thank S. Soltanian and S. H. Zhou for their help in the experiment. This work was supported by the Australian Research Council, Hyper Tech Research Inc. OH USA, Alphatech International Ltd., NZ and the University of Wollongong. W.K.Y. received an Australia-Asia Award funded by the Australian Government.

${ }^{1}$ T. Takenobu, T. Ito, Dam Hieu Chi, K. Prassides, and Y. Iwasa, Phys. Rev. B 64, 134513 (2001).

${ }^{2}$ M. Paranthaman, J. F. Thompson, D. K. Christen, Physica C 355, 1 (2001).

${ }^{3}$ A. Bharathi, S. Jemina Balaselvi, S. Kalavathi, G. L. N. Reddy, V. Sankara Sastry, Y. Haritharan, and T. S. Radhakrishnan, Physica C 370, 211 (2002).

${ }^{4}$ J. S. Ahn and E. J. Choi, cond-mat/0103169.

${ }^{5}$ I. Maurin, S. Margadonna, K. Prassides, T. Takenobu, T. Ito, D. H. Chi, Y. Iwasa, and A. Fitch, Physica B 318, 392 (2002).

${ }^{6}$ I. Maurin, S. Margadonna, K. Prassides, T. Takenobu, Y. Iwasa, and A. N. Fitch, Chem. Mater. 14, 3894 (2002).

${ }^{7}$ W. Mickelson, J. Cumings, W. Q. Han, and A. Zettl, Phys. Rev. B 65, 052505 (2002).

${ }^{8}$ Zhao-hua Cheng, Bao-gen Shen, Jian Zhang, Shao-ying Zhang, Tong-yun Zhao, and Hong-Wu Zhao, J. Appl. Phys. 91, 7125 (2002).

${ }^{9}$ R. A. Ribeiro, S. Bud'ko, C. Petrovic, and P. C. Canfield, Physica C 382, 166 (2002).

${ }^{10}$ M. Avdeev, J. D. Jorgensen, R. A. Ribeiro, S. L. Bud'ko, and P. C. Canfield, Physica C 387, 301 (2003).

${ }^{11}$ S. Lee, T. Masui, A. Yamamoto, H. Uchiyama, and S. Tajima, cond-mat/ 0305485 .

${ }^{12}$ S. X. Dou, S. Soltanian, J. Horvat, X. L. Wang, P. Munroe, S. H. Zhou, M. Ionescu, H. K. Liu, and M. Tomsic, Appl. Phys. Lett. 81, 3419 (2002).

${ }^{13}$ S. Soltanian, J. Horvat, X. L. Wang, P. Munroe, and S. X. Dou, Physica C 390, 185 (2003).

${ }^{14}$ C. H. Cheng, H. Zhang, Y. Zhao, Y. Feng, X. F. Rui, P. Munroe, H. M. Zeng, N. Koshizuka, and M. Murakami, cond-mat/0302202.

${ }^{15}$ J. Wei, Y. Li, C. Xu, B. Wei, and D. Wu, Chem. Phys. 78, 785 (2003).

${ }^{16}$ S. X. Dou, X. L. Wang, J. Horvat, D. Milliken, A. H. Li, K. Konstantinov, E. W. Collings, M. D. Sumption, and H. K. Liu, Physica C 361, 79 (2001).

${ }^{17}$ X. L. Wang, S. Soltanian, J. Horvat, A. H. Li, M. J. Qin, H. K. Liu, and S. X. Dou, Physica C 361, 149 (2001).

${ }^{18}$ S. X. Dou, V. Braccini, S. Soltanian, R. Klie, Y. Zhu, S. Li, X. L. Wang, and D. Larbalestier (unpublished). 